\section{Case Reports in Oncology}

The Author(s)

Published by S. Karger AG, Base www.karger.com/cro

\title{
Liquid Biopsy Prevents Inaccurate Her2 Status Determination by in situ Hybridization in a Patient with Invasive Ductal Adenocarcinoma of the Breast: Case Report
}

\author{
Yen-Dun Tony Tzeng ${ }^{a, b}$ Shih-En Chang ${ }^{c} \quad$ Rui Mei $^{d} \quad$ Manana Javey ${ }^{d}$ \\ ${ }^{a}$ Department of Surgery, Division of General Surgery, Kaohsiung Veterans General \\ Hospital, Kaohsiung City, Taiwan; ${ }^{b}$ Institute of Clinical Medicine, National Yang-Ming \\ University, Taipei, Taiwan; ${ }^{\mathrm{C} C e l l M a x}$ Taiwan Co. Ltd., Taipei, Taiwan; ${ }^{\mathrm{d} C e l l M a x ~ I n c .,}$ \\ Sunnyvale, CA, USA
}

\section{Keywords}

HER2 $\cdot$ Breast cancer $\cdot$ Liquid biopsy $\cdot$ Personalized therapy $\cdot$ Oncolbx

\begin{abstract}
Utilization of circulating tumor DNA as a novel and noninvasive test for diagnosis confirmation, therapy selection, and cancer surveillance is a rapidly growing area of interest. In the wake of FDA approval of a liquid biopsy test, it is important for clinicians to acknowledge the obvious clinical utility of liquid biopsy for cancer management throughout the course of the disease. This case report describes a female with invasive ductal adenocarcinoma of the breast, where liquid biopsy was instrumental for her cancer characterization and personalized therapy selection.

(C) 2017 The Author(s)


 Oncology}

\section{Introduction}

The breast cancer incidence has been reported to be up to 1.7 million annually across the globe [1]. Breast cancer is a heterogeneous group of neoplasms and is one of the few tumor types in which molecular classification has successfully been used to design and personalize therapies, leading to major improvements in survival.

HER2 gene amplification, hence protein overexpression, is present in approximately 15$20 \%$ of breast tumors [2]. HER2 amplification usually appears first in atypical ductal hyperplasia and ductal carcinoma in situ [3], and is associated with comedocarcinoma and aggressive invasive cancers. The most attractive component of this marker is that it qualifies patients for anti-HER2 treatment. Anti-HER2 therapy (e.g., trastuzumab) in combination with chemotherapy has been shown to reduce recurrence, metastases, and mortality [4, 5], as well as improve survival in metastatic disease $[6,7]$.

Given the impact that anti-HER2 treatment may have on patients, according to NCCN as well as CAP/ASCO recommendations, HER2 testing must be performed on every primary invasive carcinoma and on a metastatic site in stage IV cases. In addition, NCCN guidelines recommend re-testing of recurring HER2-negative patients for HER2 status conversion from negative to positive. Eligibility to anti-HER2 agents strictly depends on the demonstration of HER2 overexpression (by immunohistochemistry) or of HER2 gene amplification by in situ hybridization (ISH) techniques such as FISH/CISH. However, approximately $20 \%$ of cases by FISH are considered as "equivocal," which may lead to the preclusion of anti-HER2-based therapy for some patients. In addition to equivocal cases, there are scenarios when HER2 is wrongly considered as negative due to either ISH assay failure or the phenomenon of tumor heterogeneity. Here we address the tumor heterogeneity phenomenon in a female with an invasive ductal adenocarcinoma of the breast. The issue became evident to us when conflicting results were obtained by liquid biopsy and ISH assay. Circulating tumor DNA sequencing results triggered revision of the negative HER2 ISH results, which led to further studies and ultimately positive ISH results for HER2 amplification.

\section{Case Report}

A 48-year-old female presented to the Kaohsiung Hospital (Taiwan) with left breast mass in 2012. The biopsy was performed to confirm invasive ductal carcinoma of the breast. Subsequently, the patient underwent modified radical mastectomy, and surgical specimen was submitted to the pathology. The patient's cancer was stage pT2N2M0, and the tumor was classified as invasive ductal adenocarcinoma with $85 \%$ of ER+ and $60 \%$ PR+ expression, and negative $H E R 2 /$ neu.

The patient refused systemic chemotherapy and was treated with tamoxifen for nearly 2 years followed by Xeloda for a year; however, her disease progressed. Subsequent excisional biopsies of left supraclavicular lymph nodes (2015) demonstrated infiltrating invasive ductal adenocarcinoma of the breast with $55 \%$ of ER+, $10 \%$ of PR expression with negative HER2 and $60 \%$ of Ki67. Given the lack of compliance and refusal to systemic intravenous chemotherapy, the patient was given gonadotropin-releasing hormone agonist with tamoxifen, followed by Navelbine for about 3 months. Four years into the course of the disease, she suffered from general malaise, developed multiple skin nodules and elevated tumor markers (CA-153: 1,230 U/mL). PET scan showed multiple neck, mediastinal, retroperitoneal lymph 
node metastases, osteolytic lesions, bilateral lung metastases, and peritoneal seeding with massive ascites (Fig. 1).

The patient was biopsied over the left chest wall, which confirmed to be invasive carcinoma of no special type with 7\% of ER expression, negative PR and "negative" HER2 by CISH. At the same time, peripheral blood was drawn for circulating tumor DNA sequencing in plasma. The sample was sent to the CellMax Life laboratory (Taipei) for the liquid biopsy test called Oncolbx, where cell-free DNA was extracted from plasma and sequenced using CellMax Life proprietary SMSEQ technology for 73 genes. The patient's liquid biopsy results were discordant with chest wall biopsy findings as there was a significant gain (approximately $\times 40$ ) in gene copy variation observed in the ERBB2/HER2 gene (Fig. 2) by the Oncolbx test conflicting with negative ISH results for HER2 amplification. In addition to ERBB2/HER2, there were amplifications found in KRAS $(\times 10)$ and FGFR2 $(\times 20)$ genes. The liquid biopsy results triggered revision of the chest wall biopsy ISH test results, and new slides from the same lesion were stained showing HER2 signal to chromosome 17 centromere signal $\geq 2.18$, with 4.85 as an average number of HER2 gene copies per cell, and assigned as positive (Fig. 3).

Based on these findings, the patient was prescribed TDM-1, which resulted in dropping of the CA-153 tumor marker from 513.4 to $268 \mathrm{U} / \mathrm{mL}$. Despite marked correction of tumor markers, the patient's cancer evolved rapidly and the patient expired after about 1 month from the anti-HER2 treatment.

\section{Discussion}

It is obvious that more superior molecular assays are needed to determine the HER2 status. Based on the promising data available on anti-HER2 regimens, this patient might have greatly benefited from anti-HER2 treatment if administered timely at an early stage.

It is clear that the metastatic lesion from the chest wall displayed intratumoral heterogeneity in this patient, and if liquid biopsy was not performed, ISH test results would not be challenged.

This case demonstrates not only intratumoral heterogeneity within one metastatic lesion, but also heterogeneity between primary and metastatic lesions, since primary tumor was reported as HER2 negative based on the ISH assay. Primary tumor tissue was not available for further examination; hence, we do not know whether the intratumoral heterogeneity was also present within the primary lesion.

This case demonstrates a significant clinical utility of alternative assays such as liquid biopsy for assessing the complete mutational profile of a tumor during the course of the disease.

Circulating tumor DNA provides crucial genetic information as a tissue biopsy but with some major advantages [8]. First, it is a mixture of tumor DNA derived from multiple tumor sites representing tumor genome broadly compared to a localized biopsy, which introduces the sampling bias. Second, peripheral blood draw is a minimally invasive procedure, allowing serial and longitudinal profiling of a tumor genome, for monitoring of tumor burden, assessment of heterogeneity as well as evaluation of response or resistance to treatment [9].

Liquid biopsy comes truly handy in a clinical setting when dealing with the intratumoral and intermetastatic tumor heterogeneity phenomenon. Tumor heterogeneity may potentially lead to an incomplete picture of the mutational profile of the malignancy, hence provide no information that is critical for planning of targeted therapy regimens if only tissue analy- 
sis is utilized [10]. Based on the sampled tissue during the course of the disease, this patient's breast cancer varied across the sites sampled for hormone receptor expression; primary tumor tested strongly positive for ER/PR at the time of diagnosis; however, subsequent biopsy of supraclavicular lymph node showed much lower expression of ER/PR and very diminished expression of the same markers in the chest wall biopsy specimen. It is possible that HER2 expression was also variable among primary and metastatic lesions; hence, tumor heterogeneity was present. Besides tumor heterogeneity, it is noteworthy to mention that these biopsies were spatially distributed during 4 years through the course of the disease, which raises the possibility that cancer had evolved and changed its mutational profile hence phenotype due to clonal evolution. This is a clear example why only sampling tissue may not be an ideal way of assessing tumor markers for therapeutic purposes during the course of the disease, and how noninvasive liquid biopsy can help to track tumor evolution and assess the complete mutational profile of primary as well as metastatic lesions at any point of the course of the disease.

\section{Statement of Ethics}

The authors have no ethical conflicts to disclose.

\section{Disclosure Statement}

Shih-En Chang, Rui Mei, and Manana Javey are employees of CellMaxlife laboratories. Dr. Yen-Dun Tony Tzeng has nothing to disclose and has not received any gifts from CellMax Life laboratory.

\section{References}

1 Ferlay J, Soerjomataram I, Dikshit R, et al: Cancer incidence and mortality worldwide: sources, methods and major patterns in GLOBOCAN 2012. Int J Cancer 2015;136:E359-E386.

2 Burstein HJ: The distinctive nature of HER2-positive breast cancers. N Engl J Med 2005;353:16521654.

-3 Xu R, Perle MA, Inghirami G, Chan W, Delgado Y, Feiner H: Amplification of Her-2/neu gene in Her$2 /$ neu-overexpressing and -nonexpressing breast carcinomas and their synchronous benign, premalignant, and metastatic lesions detected by FISH in archival material. Mod Pathol 2002;15:116124.

4 Subramanian A, Mokbel K: The role of Herceptin in early breast cancer. Int Semin Surg Oncol 2008;5:9.

5 Paluch-Shimon S, Wolf I, Goldberg H, Evron E, Papa MZ, Shabtai M, Barsuk D, Yosepovich A, Modiano T, Catane R, Kaufman B: High efficacy of pre-operative trastuzumab combined with paclitaxel following doxorubicin and cyclophosphamide in operable breast cancer. Acta Oncol 2008;47:1564-1569.

-6 Church DN, Modgil R, Guglani S, Bahl A, Hopkins K, Braybrooke JP, Blair P, Price CG: Extended survival in women with brain metastases from HER2 overexpressing breast cancer. Am J Clin Oncol 2008;31:250-254.

7 Hayes DF, Thor AD, Dressler LG, Weaver D, Edgerton S, Cowan D, Broadwater G, Goldstein LJ, Martino S, Ingle JN, Henderson IC, Norton L, Winer EP, Hudis CA, Ellis MJ, Berry DA: HER2 and response to paclitaxel in node-positive breast cancer. Cancer and Leukemia Group B (CALGB) Investigators. N Engl J Med 2007;357:1496-106.

-8 Diaz LA Jr, Bardelli A: Liquid biopsies: genotyping circulating tumor DNA. J Clin Oncol 2014;32:579_ 586. 


\section{Case Reports in Oncology}

-9 Perkins G, Yap TA, Pope L, Cassidy AM, Dukes JP, Riisnaes R, Massard C, Cassier PA, Miranda S, Clark J, et al: Multi-purpose utility of circulating plasma DNA testing in patients with advanced cancers. PLoS One 2012;7:e47020.

-10 Gerlinger M, Rowan A.J, Horswell S, Larkin J, Endesfelder D, Gronroos E, Martinez P, Matthews N, Stewart A, Tarpey P, et al: Intratumor heterogeneity and branched evolution revealed by multiregion sequencing. N Engl J Med 2012;366:883-892.

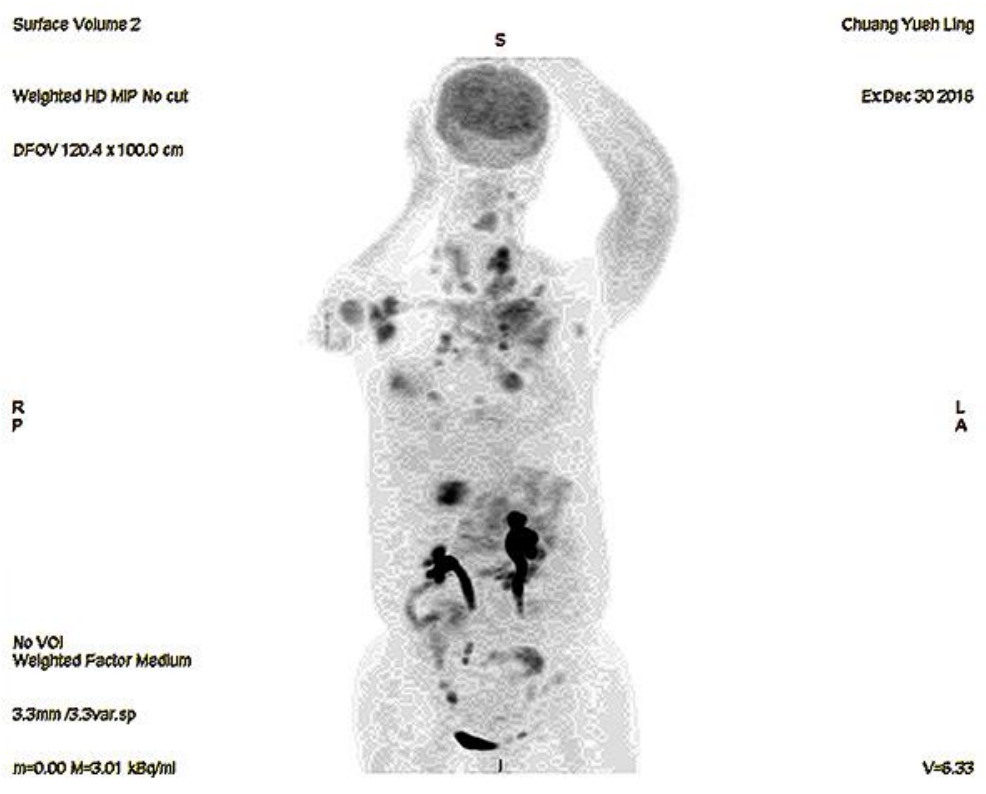

Fig. 1. The ${ }^{18} \mathrm{~F}$ fluorodeoxyglucose (FDG) PET scan from the head to pelvis revealed FDG uptake in the midline-to-left anterior chest wall, left pleura with effusion; metastatic lymph nodes in bilateral neck regions, bilateral axillary chains, left internal mammary chain, mediastinum/bihilar regions, and retroperitoneum; osteolytic metastases in C3, C4, T10; bilateral lung metastases; peritoneal/abdominal wall seeding with massive ascites. 


\section{Case Reports in Oncology}

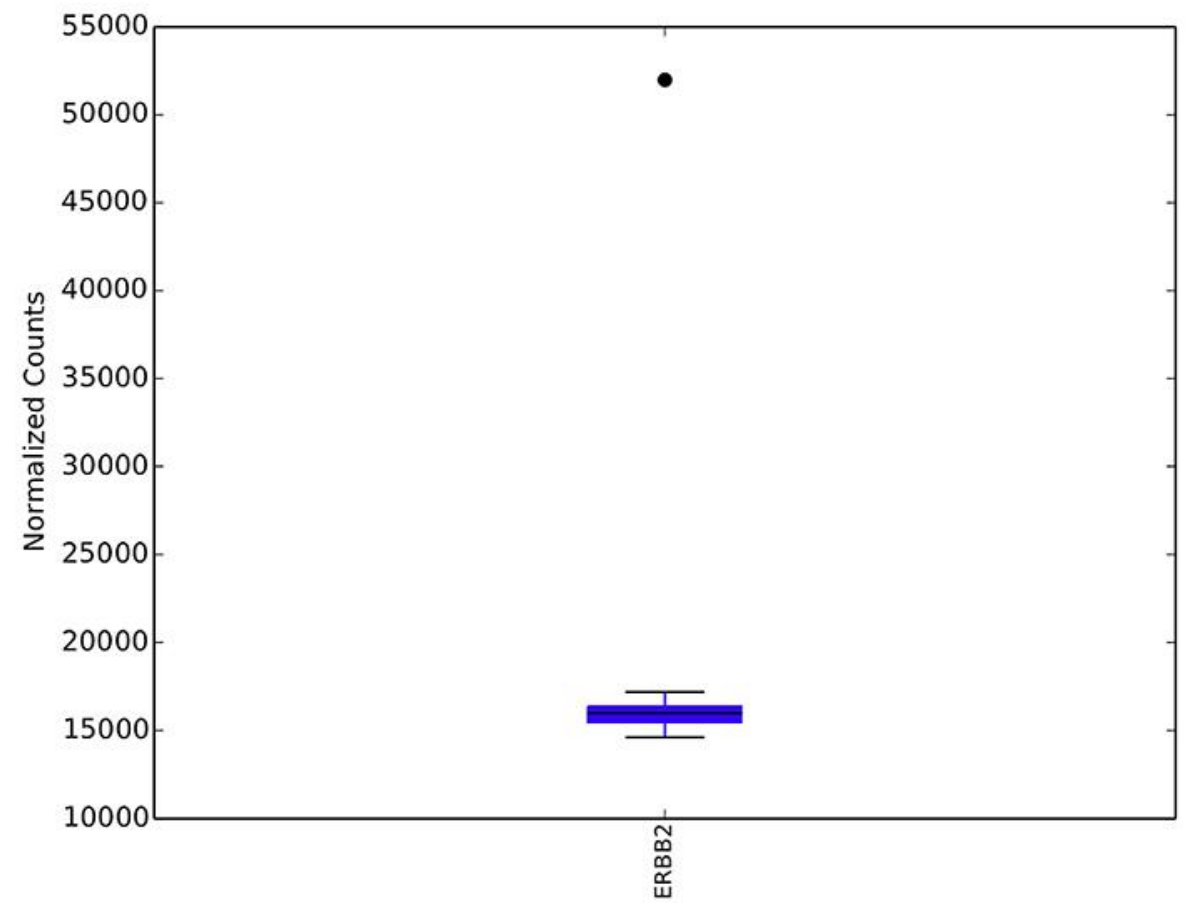

Fig. 2. Comparison between expected normal values of ERBB2 gene copies $(10,000-20,000)$ versus significantly increased observed counts of ERBB2 gene $(\sim 50,000)$ found in this patient's plasma using single molecular sequencing technology (SMSEQ).

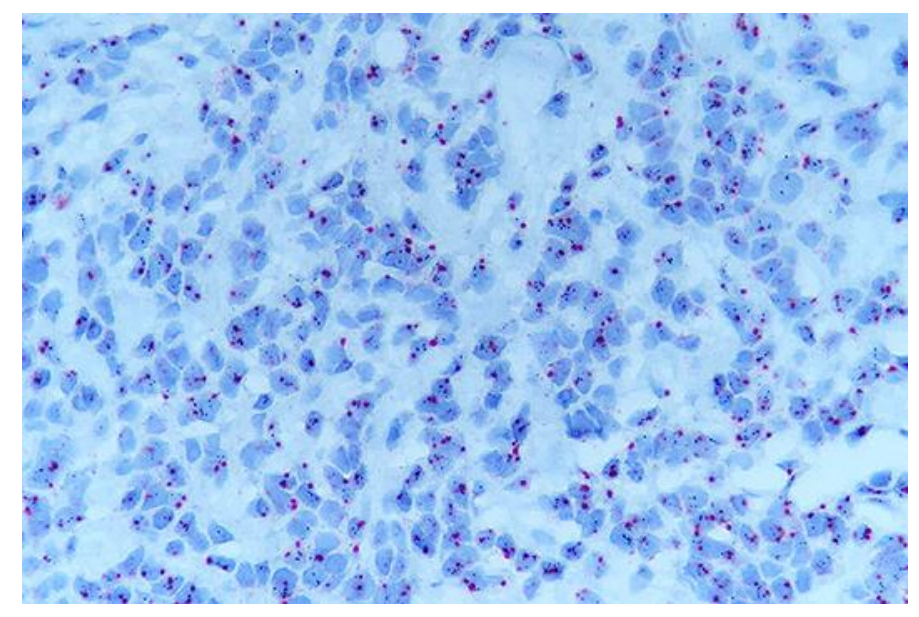

Fig. 3. This is a representative image of Her2 chromogenic in situ hybridization test performed on the chest wall biopsy. Average number of HER2 gene copies per cell observed: 4.85. Average copy number of chromosome 17 per cell: 2.25. HER2/CEP17 ratio: 2.18. The ratio of HER2 signal to chromosome 17 centromere signal observed $\geq 2.0$, hence HER2 gene amplification is considered positive. 